\title{
MinPROMEP: Generation of Partially Replicated Minimal Orthogonal Main-effect Plans Using a Novel Algorithm
}

\author{
CHWEN-MING CHANG \\ National Kaohsiung First University of Science and Technology \\ Taiwan
}

\begin{abstract}
MinPROMEP, written in SAS macros, is a program that generates partially replicated minimal orthogonal main-effect plans (minimal PROMEP's) for a given set of specified factor levels. The proposed algorithm is based on a generalization of the construction of Jacroux (1992, 1993). By using specific collapsing schemes, duplicated points are embedded in the design. In some cases, the generated minimal PROMEP has maximum number of duplicate points.
\end{abstract}

\section{$1 \quad$ Introduction}

Orthogonal main-effect plans (OMEP's) are effectively applied in the early stage in experiments to locate significant factors in many applications. As stated by Lewis and John (1976), orthogonality stands for the uncorrelated property of the estimates of all main effects in the plan while testing for hypotheses of weighted means. Under such considerations, proportional frequencies are both sufficient and necessary conditions of an OMEP according to Plackett (1946) and Addelman (1962). As noted by Jacroux (1992), partially replicated minimal orthogonal main-effect plans (minimal PROMEP's) are OMEP's with two additional properties: (1) Their run numbers reach the lower bounds 
of all possible OMEP's with specified factor levels. (2) They are constructed so that a certain number of duplicate points are obtained yet the orthogonality of the plans is kept. The advantages of minimal PROMEP's are obvious. First, this reduces the cost of experiments since the plans have the least run numbers among OMEP's. Second, partial replication facilitates the analysis of experimental data as indicated by Pigeon and McAllister (1989). The pure error can be estimated without assuming the model.

To check the existence of Property (1), Jacroux (1992) provides the sufficient conditions for an OMEP having the desired number of factors and specified levels with a minimal number of runs. Theorem 1 reproduces the sufficient conditions.

Theorem 1 (Jacroux, 1992) Suppose that an OMEP d has $k \geq 3$ factors in which factor $i$ has $s_{i}$ levels, $i=1, \ldots, k, s_{1} \geq s_{2} \geq \cdots \geq s_{k}$ and $N$ runs. If $N=\tilde{s}_{1} \tilde{s}_{2}$ for $\tilde{s}_{1} \geq s_{1}$ and $\tilde{s}_{2} \geq s_{2}$ satisfying

$$
\tilde{s}_{1} \tilde{s}_{2}=\min _{x \geq s_{1}, y \geq s_{2}} x y<2 s_{1} s_{2}, \quad s_{3}\{\operatorname{lcm}(x, y)\} \leq x y
$$

where $\operatorname{lcm}(x, y)$ denotes the least common multiple between positive integers $x$ and $y$, then d is a minimal OMEP.

Property (2) can be obtained through processes such as the interchange algorithm, special arrangements of the plans, and the utilization of an appropriate collapsing scheme (e.g., see Burgess and Street (1994), Burgess and Street (1999), Chang (1998), Jacroux (1992, 1993), Street (1994)). The appropriate collapsing scheme is a simple and rapid means of ensuring a certain number of duplicate points in the plan. Herein, "collapsing" is a common technique used during the construction of OMEP's. Assume that $m_{1}>m_{2}$, an $m_{1}$-level factor can be collapsed to an $m_{2}$-level factor by applying many-to-one mapping from the set of $m_{1}$ levels into the set of $m_{2}$ levels. Addelman (1962) verifies that the 
orthogonality of the plan is maintained during collapsing. Under the usage of proper collapsing schemes, Chang (2003) further develops a generalization of the construction of Jacroux (1992). In particular, the restriction of $\tilde{s}_{1}$ being a multiple of $\tilde{s}_{2}$ is removed. This is the construction method adopted by MinPROMEP. In next section, the method is introduced. In Section 3, the program algorithm is described. Finally, the using of MinPROMEP is demonstrated and illustrative examples are given.

\section{The Construction Methods in MinPROMEP}

Two major theorems used in MinPROMEP are restated as the follows (Chang, 2003).

Theorem 2 (Chang, 2003) Assume that $\tilde{s}_{1} \geq \tilde{s}_{2}$ satisfies the sufficient conditions of Theorem 1 and $\tilde{s}_{3}$ equals $\operatorname{gcd}\left(\tilde{s}_{1}, \tilde{s}_{2}\right)$. Assume further that $q$ orthogonal latin squares of size $\tilde{s}_{3} \times \tilde{s}_{3}$ exist. An $\tilde{s}_{1} \times \tilde{s}_{2} \times t_{1} \times \cdots \times t_{q} / / \tilde{s}_{1} \tilde{s}_{2}$ minimal OMEP $D$ can be constructed for $q+2$ factors where $t_{1}=\cdots=t_{q}=\tilde{s}_{3}$.

Theorem 3 (Chang, 2003) Assume that $\tilde{s}_{1} \geq \tilde{s}_{2}$ satisfies the sufficient conditions of Theorem 1 and $\tilde{s}_{3}$ equals $\operatorname{gcd}\left(\tilde{s}_{1}, \tilde{s}_{2}\right)$. Assume also that there exists $q$ orthogonal latin squares of size $\tilde{s}_{3} \times \tilde{s}_{3}$. A minimal OMEP $d$ with partial replication can be constructed for $q+2$ factors with factor level number $s_{1} \geq s_{2} \geq \cdots \geq s_{q+2}, s_{1} \leq \tilde{s}_{1}, s_{2} \leq \tilde{s}_{2}$ and $s_{3} \leq \tilde{s}_{3}$.

Class 1: If $\tilde{s}_{1}>s_{1}, \tilde{s}_{2}=s_{2}$, a minimal OMEP with $\left(\tilde{s}_{1}-s_{1}\right) \times \tilde{s}_{2}$ duplicate points can be obtained by appropriately collapsing $\tilde{s}_{1}$ levels offactor 1 to $s_{1}$ levels;

Class 2: If $\tilde{s}_{1}=s_{1}, \tilde{s}_{2}>s_{2}$, a minimal OMEP with a certain number of duplicate points can be obtained by appropriately collapsing the $\tilde{s}_{2}$ levels of factor 2 to $s_{2}$ levels: 
$\tilde{s}_{1} \times\left(\tilde{s}_{2}-s_{2}\right)$ duplicate points can be obtained if $s_{2}>\tilde{s}_{3}$ and $\left(\tilde{s}_{1} / \tilde{s}_{2}\right) \times\left(\tilde{s}_{2}-s_{2}\right)$ duplicate points can be obtained if $s_{2} \leq \tilde{s}_{3}$;

Class 3: If $\tilde{s}_{1}>s_{1}, \tilde{s}_{2}>s_{2}$, a minimal OMEP with a certain number of duplicate points can be obtained by appropriately collapsing $\tilde{s}_{1}$ levels of factor 1 to $s_{1}$ levels, and $\tilde{s}_{2}$ levels of factor 2 to $s_{2}$ levels: $\left(\tilde{s}_{1}-s_{1}\right) \times s_{2}+\tilde{s}_{1} \times\left(\tilde{s}_{2}-s_{2}\right)$ duplicate points can be obtained if $s_{2}>\tilde{s}_{3}$ and $\left(\tilde{s}_{1} / \tilde{s}_{2}\right) \times\left(\tilde{s}_{2}-s_{2}\right)+\left(\tilde{s}_{1}-s_{1}\right) \times \tilde{s}_{2}$ duplicate points can be obtained if $s_{2} \leq \tilde{s}_{3}$;

Class 4: If $\tilde{s}_{1}=s_{1}, \tilde{s}_{2}=s_{2}$, a minimal OMEP cannot have any duplicate points.

In fact, Theorem 3 ensures that maximum number of duplicate points are obtained when (1) $\tilde{s}_{1}>s_{1}$, and $\tilde{s}_{2}=s_{2}$, (2) $\tilde{s}_{1}=s_{1}$ and $\tilde{s}_{2}>s_{2}>\tilde{s}_{3}$, and (3) $\tilde{s}_{1}>s_{1}$ and $\tilde{s}_{2}>s_{2}>\tilde{s}_{3}$.

The algorithm developed here also considers the case of three-factor minimal PROMEP's for factor levels $s_{1} \times\left(s_{1}-1\right)^{2}$ when $s_{1} \geq 5$. A related corollary is reproduced in the following. The constructed minimal PROMEP contains maximum number of duplicate points.

Corollary 1 (Chang, 1998) A three-factor OMEP having levels of factors $s_{1} \times\left(s_{1}-1\right)^{2}$ exists with minimal runs and maximum duplicate points for $s_{1} \geq 5$.

\section{$3 \quad$ Description of the Proposed Algorithm}

The largest three factor levels $-s_{1}, s_{2}$ and $s_{3}$ as well as the name of the dataset storing the plan are the required input. By receiving the specified factor levels $-s_{1}$ and $s_{2}$, $\tilde{s}_{1}$ and $\tilde{s}_{2}$ are then calculated according to Theorem 1. Set $\tilde{s}_{3}$ as the greatest common divisor of $\tilde{s}_{1}$ and $\tilde{s}_{2}$. Next, a minimal OMEP with $\tilde{s}_{3}+1$ factors is generated if $\tilde{s}_{3}$ is 
a prime or a power of a prime. Otherwise, a three-factor minimal PROMEP is generated. The output dataset contains a minimal PROMEP, $s_{1} \times s_{2} \times s_{3}{ }^{k-2} / / \tilde{s}_{1} \tilde{s}_{2}$, with a certain number of duplicate points. Herein, $s_{1} \times s_{2} \times s_{3}{ }^{k-2} / / \tilde{s}_{1} \tilde{s}_{2}$ represents an OMEP having one $s_{1}$-level factor, one $s_{2}$-level factor and $k-2$ factors of $s_{3}$ level in $\tilde{s}_{1} \tilde{s}_{2}$ runs. Moreover, when $s_{2}=s_{3}=s_{1}-1$ and $s_{1} \geq 5$, a three-factor minimal PROMEP with maximum number of duplicate points is also generated according to Corollary 1.

In this program, two constraints are imposed for the parameters: (a) $s_{3} \geq 2$, and (b) $\tilde{s}_{3} \leq 20$.

The following describes the algorithm in steps.

Step 1 When $s_{2}=s_{3}=s_{1}-1$ and $s_{1} \geq 5$, a three-factor minimal PROMEP with maximum duplicate points is generated according to Corollary 1. Print out the plan.

Step 2 Calculate $\tilde{s}_{1}$ and $\tilde{s}_{2}$ according to Theorem 1. Calculate $\tilde{s}_{3}$.

$\underline{\text { Step } 3}$ If $\tilde{s}_{3}$ is a prime or a power of a prime, generate the minimal OMEP $d_{0}$ in $\tilde{s}_{3}+1$ factors; otherwise, generate the minimal OMEP $d_{0}$ in three factors.

Step 4 Collapse factor 1 into the $s_{1}$ level, and factor 2 into the $s_{2}$ level as described in the proof of Theorem 3 for design $d_{0}$. Collapse the rest of the factors into $s_{3}$ level.

Step 5 Print out the generated minimal PROMEP.

\section{The operation of MinPROMEP}

Assume that file "all.sas" contains all macros and is inserted from disk driver "A:l". Since major macro is named "MinPROMEP.sas" in this study, type in the commands in the SAS environment as follows.

\%include "A:\all.sas; 
$\% \operatorname{MinPROMEP}\left(s_{1}, s_{2}, s_{3}, d s n\right)$;

Here, $s_{1}, s_{2}$ and $s_{3}$ are the first three largest factor levels and $d s n$ is the desired dataset name for output. The output includes the generated plan and the number of duplicated points produced. If the maximum number of duplicate points for the plan is reached, a confirmation remark is given in the footnote.

\section{$5 \quad$ Illustrative Examples}

In the following, five examples are given for various classes of plans in Theorem 3. Moreover, Example 1 constructs the design by Corollary 1. Example 5 demonstrates the construction when $\widetilde{s}_{3}$ is neither a prime nor a power of a prime.

Example 1: Submit the following commands

$$
\begin{aligned}
& \text { \%include "A: \all.sas"; } \\
& \text { \%MinPROMEP(5, 4, 4, dsn1); }
\end{aligned}
$$

Then, two plans are generated and stored in two datasets. One is the $5 \times 4^{2} / / 25$ minimal PROMEP with maximum number of duplicate points by Corollary 1 . The plan is stored in the file named by an extra "A" in front of user specified dataset name. The other is the $5 \times 4^{5} / / 49$ minimal PROMEP with one duplicate point by Theorem 3. See Output Listing 1(a) and 1(b) for the two plans.

\section{Output Listing 1(a):}

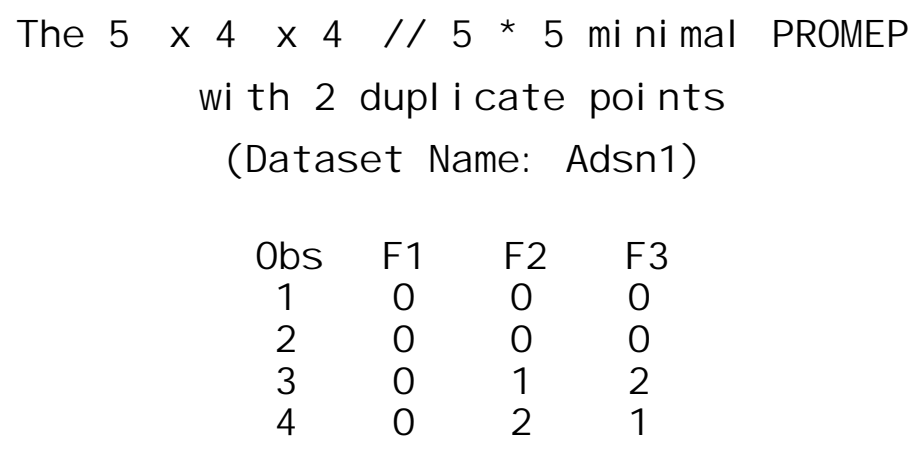




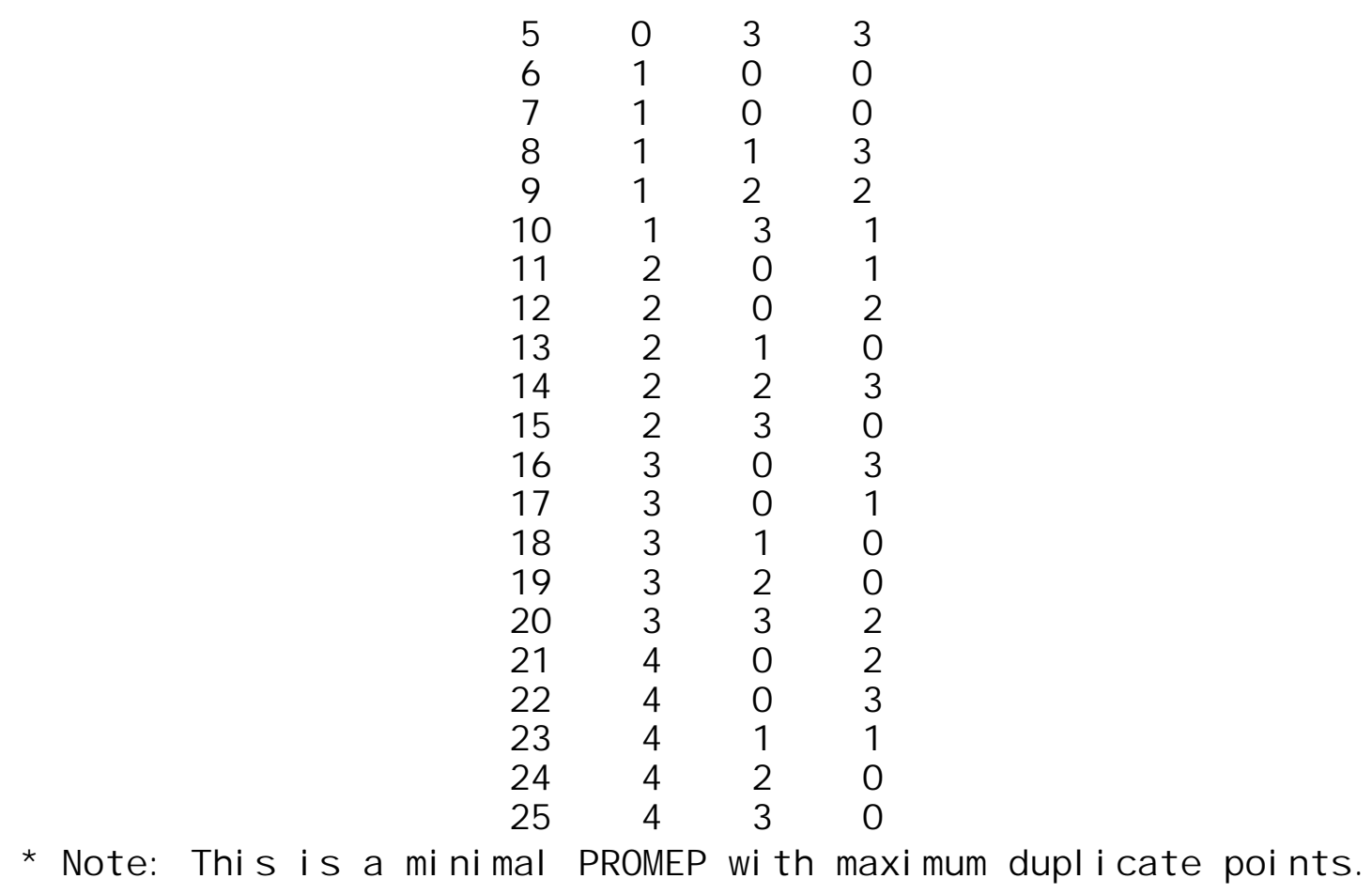

Output Listing 1(b):

$$
\begin{gathered}
\text { The } 5 \times 4 \times 4 \wedge 4 / / 5 * 5 \text { minimal PROMEP } \\
\text { with } 1 \text { duplicate points } \\
\text { (Dataset Name: dsn1) }
\end{gathered}
$$

$\begin{array}{ccccccc}\text { Obs } & \text { F 1 } & \text { F2 } & \text { F 3 } & \text { F4 } & \text { F5 } & \text { F 6 } \\ 1 & 0 & 0 & 0 & 0 & 0 & 0 \\ 2 & 0 & 0 & 0 & 0 & 0 & 0 \\ 3 & 0 & 1 & 1 & 1 & 1 & 1 \\ 4 & 0 & 2 & 2 & 2 & 2 & 2 \\ 5 & 0 & 3 & 3 & 3 & 3 & 3 \\ 6 & 1 & 0 & 0 & 1 & 2 & 3 \\ 7 & 1 & 0 & 1 & 2 & 3 & 0 \\ 8 & 1 & 1 & 2 & 3 & 0 & 0 \\ 9 & 1 & 2 & 3 & 0 & 0 & 1 \\ 10 & 1 & 3 & 0 & 0 & 1 & 2 \\ 11 & 2 & 0 & 1 & 3 & 0 & 2 \\ 12 & 2 & 0 & 2 & 0 & 1 & 3 \\ 13 & 2 & 1 & 3 & 0 & 2 & 0 \\ 14 & 2 & 2 & 0 & 1 & 3 & 0 \\ 15 & 2 & 3 & 0 & 2 & 0 & 1 \\ 16 & 3 & 0 & 2 & 0 & 3 & 1 \\ 17 & 3 & 0 & 3 & 1 & 0 & 2 \\ 18 & 3 & 1 & 0 & 2 & 0 & 3 \\ 19 & 3 & 2 & 0 & 3 & 1 & 0 \\ 20 & 3 & 3 & 1 & 0 & 2 & 0 \\ 21 & 4 & 0 & 0 & 3 & 2 & 1 \\ 22 & 4 & 0 & 3 & 2 & 1 & 0\end{array}$




$$
\begin{array}{lllllll}
23 & 4 & 1 & 0 & 0 & 3 & 2 \\
24 & 4 & 2 & 1 & 0 & 0 & 3 \\
25 & 4 & 3 & 2 & 1 & 0 & 0
\end{array}
$$

Example 2: Submit the following commands

$$
\begin{aligned}
& \text { \%include “A:\all.sas"; } \\
& \text { \%MinPROMEP(7, 4, 4, dsn2); }
\end{aligned}
$$

Since $\tilde{s}_{1}=8, \tilde{s}_{2}=4$, this is a case of $\underline{\text { Class } 1}$ in Theorem 3 that $\tilde{s}_{1}>s_{1}, \tilde{s}_{2}=s_{2}$. The $7 \times 4^{4} / / 32$ minimal PROMEP with 4 duplicate points is generated. The plan obtains maximum number of duplicate points. See Output Listing 2 for the plan.

\section{Output Listing 2:}

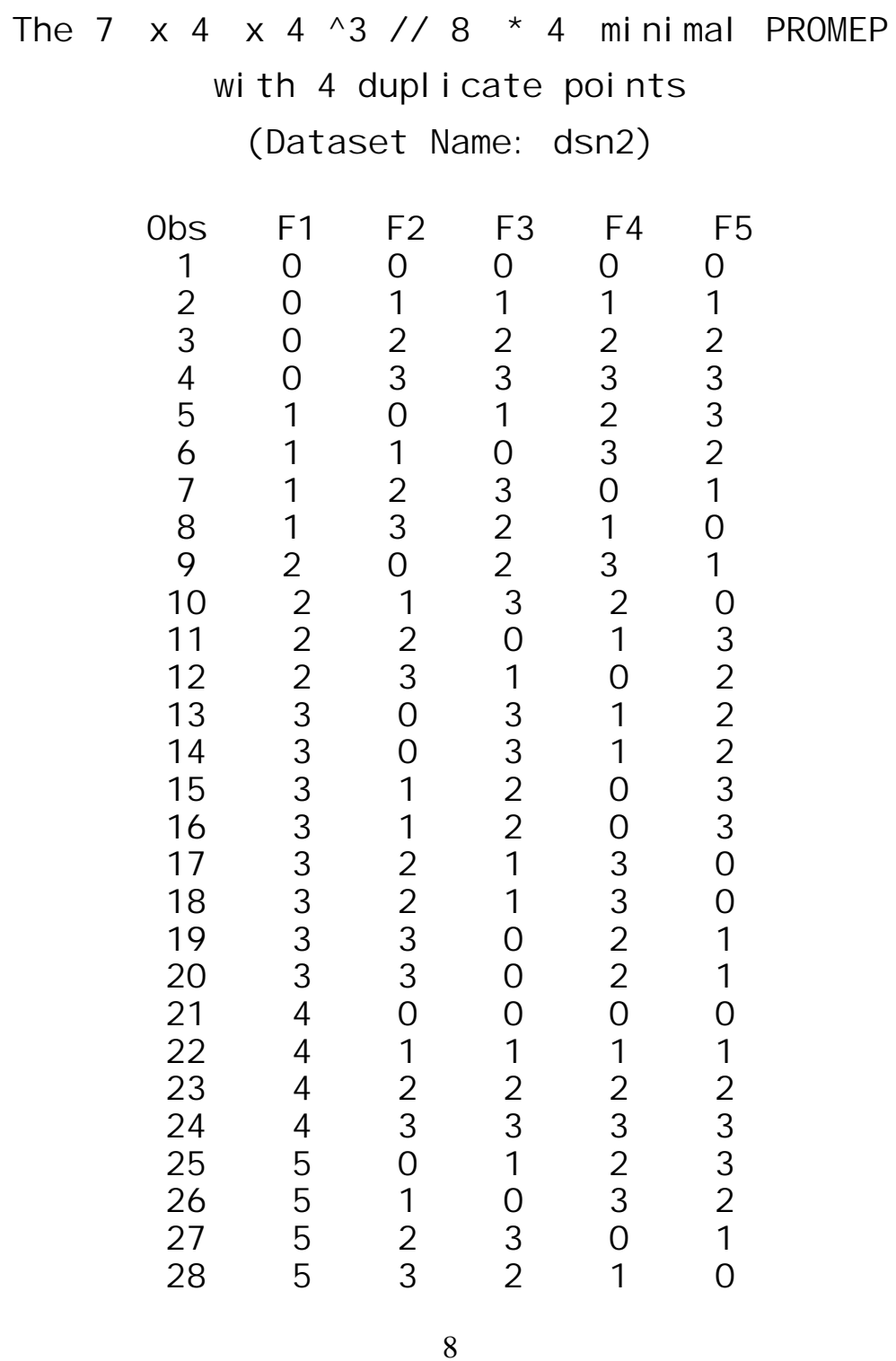




$\begin{array}{llllll}29 & 6 & 0 & 2 & 3 & 1 \\ 30 & 6 & 1 & 3 & 2 & 0 \\ 31 & 6 & 2 & 0 & 1 & 3 \\ 32 & 6 & 3 & 1 & 0 & 2\end{array}$

* Note: This is a mi nimal PROMEP with maximum duplicate points.

Example 3: Submit the following commands

\%include "A:\all.sas";

\%MinPROMEP(4, 3, 3, dsn3);

Since $\tilde{s}_{1}=4, \tilde{s}_{2}=4$, this is a case of Class 2 in Thoerem 3 that $\tilde{s}_{1}=s_{1}, \tilde{s}_{2}>s_{2}$ and $s_{2}<\tilde{s}_{3}$. The plan generates $4 \times 3 \times 3^{3} / / 16$ minimal PROMEP with 1 duplicate point. It can be verified that the maximal number of duplicate points for plan $4 \times 3^{4} / / 16$ equals 1 . See Output Listing 3 for the plan.

\section{Output Listing 3:}

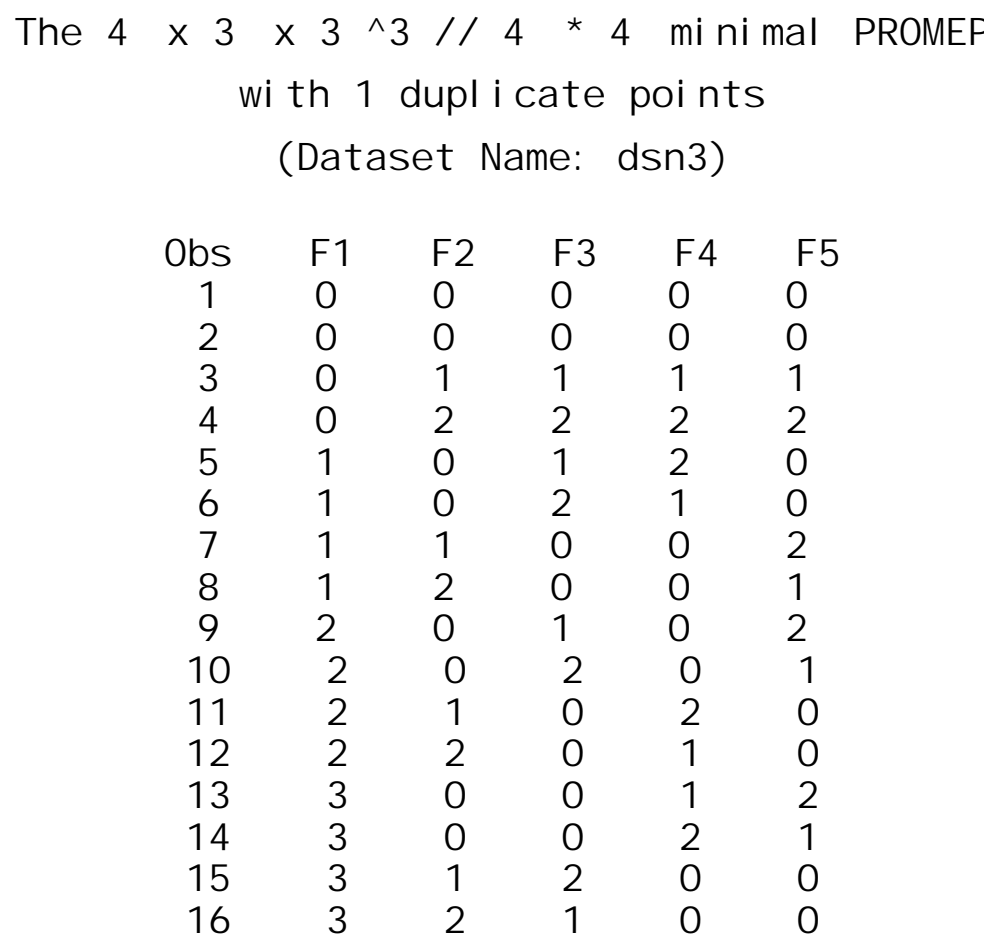

Example 4: Submit the following commands

\%include “A:\all.sas"; 


\section{\%MinPROMEP(11, 7, 3, dsn4);}

Since $\tilde{s}_{1}=12, \widetilde{s}_{2}=8$, this is a case of $\underline{\text { Class } 3}$ in Theorem 3 that $\widetilde{s}_{1}>s_{1}, \widetilde{s}_{2}>s_{2}$ and $s_{2}>\tilde{s}_{3}$. The plan generates $11 \times 7 \times 3^{3} / / 96$ minimal PROMEP with the maximum of 19 duplicate points. See Output Listing 4 for the plan.

\section{Output Listing 4:}

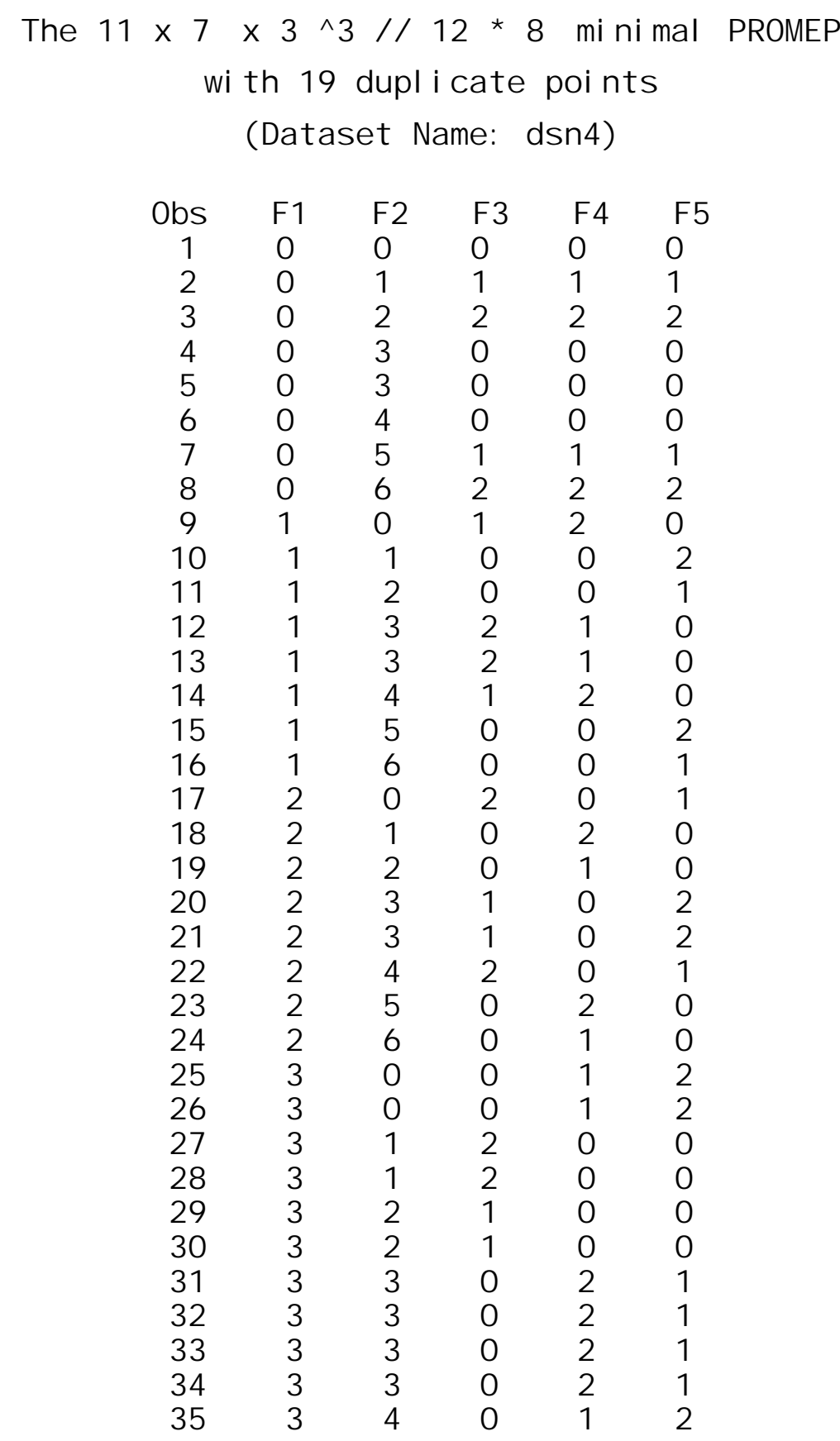




\begin{tabular}{|c|c|c|c|c|}
\hline 36 & 3 & 4 & 0 & 1 \\
\hline 37 & 3 & 5 & 2 & 0 \\
\hline 38 & 3 & 5 & 2 & 0 \\
\hline 39 & 3 & 6 & 1 & 0 \\
\hline 40 & 3 & 6 & 1 & 0 \\
\hline 41 & 4 & 0 & 0 & 0 \\
\hline 42 & 4 & 1 & 1 & 1 \\
\hline 43 & 4 & 2 & 2 & 2 \\
\hline 44 & 4 & 3 & 0 & 0 \\
\hline 45 & 4 & 3 & 0 & 0 \\
\hline 46 & 4 & 4 & 0 & 0 \\
\hline 47 & 4 & 5 & 1 & 1 \\
\hline 48 & 4 & 6 & 2 & 2 \\
\hline 49 & 5 & 0 & 1 & 2 \\
\hline 50 & 5 & 1 & 0 & 0 \\
\hline 51 & 5 & 2 & 0 & 0 \\
\hline 52 & 5 & 3 & 2 & 1 \\
\hline 53 & 5 & 3 & 2 & 1 \\
\hline 54 & 5 & 4 & 1 & 2 \\
\hline 55 & 5 & 5 & 0 & 0 \\
\hline 56 & 5 & 6 & 0 & 0 \\
\hline 57 & 6 & 0 & 2 & 0 \\
\hline 58 & 6 & 1 & 0 & 2 \\
\hline 59 & 6 & 2 & 0 & 1 \\
\hline 60 & 6 & 3 & 1 & 0 \\
\hline 61 & 6 & 3 & 1 & 0 \\
\hline 62 & 6 & 4 & 2 & 0 \\
\hline 63 & 6 & 5 & 0 & 2 \\
\hline 64 & 6 & 6 & 0 & 1 \\
\hline 65 & 7 & 0 & 0 & 1 \\
\hline 66 & 7 & 1 & 2 & 0 \\
\hline 67 & 7 & 2 & 1 & 0 \\
\hline 68 & 7 & 3 & 0 & 2 \\
\hline 69 & 7 & 3 & 0 & 2 \\
\hline 70 & 7 & 4 & 0 & 1 \\
\hline 71 & 7 & 5 & 2 & 0 \\
\hline 72 & 7 & 6 & 1 & 0 \\
\hline 73 & 8 & 0 & 0 & 0 \\
\hline 74 & 8 & 1 & 1 & 1 \\
\hline 75 & 8 & 2 & 2 & 2 \\
\hline 76 & 8 & 3 & 0 & 0 \\
\hline 77 & 8 & 3 & 0 & 0 \\
\hline 78 & 8 & 4 & 0 & 0 \\
\hline 79 & 8 & 5 & 1 & 1 \\
\hline 80 & 8 & 6 & 2 & 2 \\
\hline 81 & 9 & 0 & 1 & 2 \\
\hline 82 & 9 & 1 & 0 & 0 \\
\hline 83 & 9 & 2 & 0 & 0 \\
\hline 84 & 9 & 3 & 2 & 1 \\
\hline 85 & 9 & 3 & 2 & 1 \\
\hline 86 & 9 & 4 & 1 & 2 \\
\hline 87 & 9 & 5 & 0 & 0 \\
\hline 88 & 9 & 6 & 0 & 0 \\
\hline 89 & 10 & 0 & 2 & 0 \\
\hline
\end{tabular}




$\begin{array}{llllll}90 & 10 & 1 & 0 & 2 & 0 \\ 91 & 10 & 2 & 0 & 1 & 0 \\ 92 & 10 & 3 & 1 & 0 & 2 \\ 93 & 10 & 3 & 1 & 0 & 2 \\ 94 & 10 & 4 & 2 & 0 & 1 \\ 95 & 10 & 5 & 0 & 2 & 0 \\ 96 & 10 & 6 & 0 & 1 & 0\end{array}$

* Note: This is a mi nimal PROMEP with maximum duplicate points.

Example 5: Submit the following commands

\%include “A:\all.sas";

$\% \operatorname{MinPROMEP}(11,6,6, d s n 5)$;

Since $\tilde{s}_{1}=12, \tilde{s}_{2}=6$, this is a case of $\underline{\text { Class } 1}$ in Theorem 3. The plan generates $11 \times 6^{2} / / 72$ minimal PROMEP with 6 duplicate points. The plan obtains maximum number of duplicate points. See Output Listing 5 for the plan.

\section{Output Listing 5:}

The $11 \times 6 \times 6 / / 12 * 6$ mi ni mal PROMEP

with 6 duplicate points

( Dat aset Name: dsn5)

$\begin{array}{cccl}\text { Obs } & F 1 & F 2 & F 3 \\ 1 & 0 & 0 & 0 \\ 2 & 0 & 1 & 1 \\ 3 & 0 & 2 & 2 \\ 4 & 0 & 3 & 3 \\ 5 & 0 & 4 & 4 \\ 6 & 0 & 5 & 5 \\ 7 & 1 & 0 & 1 \\ 8 & 1 & 1 & 2 \\ 9 & 1 & 2 & 3 \\ 10 & 1 & 3 & 4 \\ 11 & 1 & 4 & 5 \\ 12 & 1 & 5 & 0 \\ 13 & 2 & 0 & 2 \\ 14 & 2 & 1 & 3 \\ 15 & 2 & 2 & 4 \\ 16 & 2 & 3 & 5 \\ 17 & 2 & 4 & 0 \\ 18 & 2 & 5 & 1 \\ 19 & 3 & 0 & 3 \\ 20 & 3 & 1 & 4 \\ 21 & 3 & 2 & 5 \\ 22 & 3 & 3 & 0 \\ 23 & 3 & 4 & 1 \\ 24 & 3 & 5 & 2\end{array}$




$\begin{array}{rrrl}25 & 4 & 0 & 4 \\ 26 & 4 & 1 & 5 \\ 27 & 4 & 2 & 0 \\ 28 & 4 & 3 & 1 \\ 29 & 4 & 4 & 2 \\ 30 & 4 & 5 & 3 \\ 31 & 5 & 0 & 5 \\ 32 & 5 & 0 & 5 \\ 33 & 5 & 1 & 0 \\ 34 & 5 & 1 & 0 \\ 35 & 5 & 2 & 1 \\ 36 & 5 & 2 & 1 \\ 37 & 5 & 3 & 2 \\ 38 & 5 & 3 & 2 \\ 39 & 5 & 4 & 3 \\ 40 & 5 & 4 & 3 \\ 41 & 5 & 5 & 4 \\ 42 & 5 & 5 & 4 \\ 43 & 6 & 0 & 0 \\ 44 & 6 & 1 & 1 \\ 45 & 6 & 2 & 2 \\ 46 & 6 & 3 & 3 \\ 47 & 6 & 4 & 4 \\ 48 & 6 & 5 & 5 \\ 49 & 7 & 0 & 1 \\ 50 & 7 & 1 & 2 \\ 51 & 7 & 2 & 3 \\ 52 & 7 & 3 & 4 \\ 53 & 7 & 4 & 5 \\ 54 & 7 & 5 & 0 \\ 55 & 8 & 0 & 2 \\ 56 & 8 & 1 & 3 \\ 57 & 8 & 2 & 4 \\ 58 & 8 & 3 & 5 \\ 59 & 8 & 4 & 0 \\ 60 & 8 & 5 & 1 \\ 61 & 9 & 0 & 3 \\ 62 & 9 & 1 & 4 \\ 63 & 9 & 2 & 5 \\ 64 & 9 & 3 & 0 \\ 65 & 9 & 4 & 1 \\ 66 & 9 & 5 & 2 \\ 67 & 10 & 0 & 4 \\ 68 & 10 & 1 & 5 \\ 69 & 10 & 2 & 0 \\ 70 & 10 & 3 & 1 \\ 71 & 10 & 4 & 2 \\ 72 & 10 & 5 & 3\end{array}$

* Note: This is a mi nimal PROMEP with maximum duplicate points. 


\section{References}

Addelman, S. (1962). "Orthogonal Main-effect Plans for Asymmetrical Factorial Experiments". Technometrics, 4, 21-46.

Burgess, L. and Street D. J. (1994). “Algorithms for Constructing Orthogonal Main Effect Plans". Utilitas Mathematica, 46, 33-48.

Burgess, L. and Street D. J. (1999). “An Interchange algorithm for Four Factor Orthogonal Main Effect Plans”. Statistics and Computing, 9, 171-177.

Chang, C.-M. (1998). "Minimal Orthogonal Main-effect Plans and Partial Replication". Journal of statistical planning and inference, 70, 167-179.

Chang, C.-M. (2003)."Construction of Minimal Orthogonal Main-effect Plans Using a General Procedure". (accepted by utilitas mathematica).

Jacroux, M. (1992). "A Note on the Determination and Construction of Minimal Orthogonal Main-effect Plans". Technometrics, 34, 92-96.

Jacroux, M. (1993). "On the Construction of Minimal Partially Replicated Orthogonal Main-effect Plans". Technometrics, 35, 32-36.

Lewis, S. M. and John J. A. (1976). “Testing Main Effects in Fractions of Asymmetrical Factorial Experiments". Biometrika, 63, 678-680.

Pigeon, J. and McAllister, P. R. (1989). "A Note on Partially Replicated Orthogonal Main-effect Plans". Technometrics, 31, 249-251.

Plackett, R. L. (1946). "Some Generalizations in the Multifactorial Design," Biometrika, $33,328-332$. 
Street, D. J. (1994). “Constructions for Orthogonal Main Effect Plans”. Utilitas Mathematica, 45, 115-123. 